\title{
Influence of Shape Elements on Performance during Haptic Rotation
}

\author{
Kathrin Krieger $^{1}$, Alexandra Moringen ${ }^{1}$ Astrid M.L. Kappers ${ }^{2}$, and Helge \\ Ritter $^{1}$ \\ 1 Neuroinformatics, CITEC, University Bielefeld, Germany \\ $\{$ kkrieger, abarch\}@techfak.uni-bielefeld.de \\ 2 Faculty of Behavioural and Movement Sciences, Department of Human Movement \\ Science, Amsterdam, The Netherlands
}

\begin{abstract}
In this work, we investigate how local shape elements of a grasped object affect performance of haptic rotation. Blindfolded participants were asked to grasp a rotary knob using thumb and index finger and to rotate it 90 degrees counterclockwise around its own axis. The knobs exhibited a suitably distributed "grasp conform" combination of local shape elements (edged, flat or round). We tested all possible scenarios where both fingers had to grasp one of the three shape elements, resulting in a total of nine experimental conditions. Based on the rotation angle, determined with a novel apparatus named Twister, we evaluated the variable and signed errors. The results imply that a round rotary knob is the hardest to rotate by a correct goal angle, while other local shape elements can benefit the performance. Independent of the experimental condition, we found a bias towards rotating too far.
\end{abstract}

Keywords: haptic rotation, rotation accuracy, local shape elements

\section{Introduction}

Humans often perform rotations by touch ${ }^{3}$, to interact with the environment in general and to control devices. To facilitate the manipulation of customer devices, there is a long tradition of non-circular shaped rotary knobs. However, haptic design is usually based on experience and far less on empirical analysis [?]. Our goal is, therefore, to investigate whether local shape elements, located under the finger tips during haptic rotation, deliver orientational cues and influence performance.

Research on haptic perception of orientations $[5,15]$ includes investigations on the oblique effect $[4,6,7,8]$ and on parallelism $[10,11,13,16]$. The oblique effect describes that vertical or horizontal orientations are better processed by humans than oblique orientations, such as 45 or 135 degrees. In parallelism experiments, participants usually explore the angular orientation of a rod and reproduce it with another one. Results show systematic deviations between the

\footnotetext{
${ }^{3}$ We refer to this rotation performed by touch only, as haptic rotation.
} 
physical parallelism and the perceived one, which can be explained by different reference frames.

Studies on human ability to achieve a given orientation that examine the oblique effect usually use reproduction tasks. Blindfolded participants have to explore a rod and reproduce its orientation after a short time delay, e.g. [6,7,8]. Other studies had participants produce verbally ordered orientations $[4,14]$. In experiments in which the perception of parallelism is investigated, e.g. [10,11, $13,16]$, participants usually have one hand on a reference bar and the other one on a test bar. Their task is to align the test bar to the reference bar. Thus, in these studies participants have to be aware of one orientation. In contrast, we examine how well humans rotate objects by a given target angle. This is a more complex task, because humans have to be aware of two orientations, start and end, or rely on knowledge about the extent of their hand rotation.
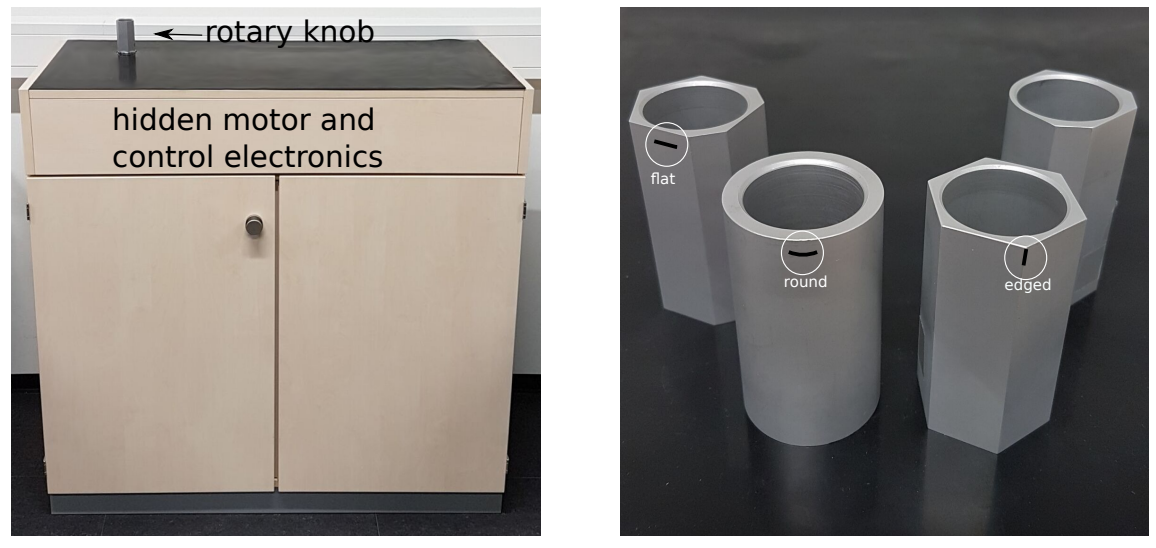

Fig. 1. Left: The Twister front view. The current design of Twister in a form of a cupboard aims to hide the extensive control electronics and motor from the participants in case the rotational joint is used actively. Participants are placed behind the cupboard during the experiments. The apparatus can be separated into two parts, the top and the bottom, in case the height needs to be adjusted manually. Right: Examples of different rotary knobs.

Previous research used rods or bars for control $[2,3,4,6,7,8,10,11,12,13$, $14,16]$, whose shape continually provides information about their orientation. In this work, participants manipulate rotary knobs, which allow us to control the information on the control's orientation as opposed to the non-uniform shape of bars. This work investigates the impact of three basic shape elements integrated locally into the rotary knobs (flat, round and edged) on the performance of target angle selection.

Fig. 1 illustrates our Twister test setup and the different rotary knobs employed. Twister will be described in detail in Section 2.2. Prior studies in this setting were in a robotic context instead of analyzing human behavior. They have focused on using haptics to extract object shape from rotational manip- 
ulations [?] or modeling the correction of pose estimates during exploratory rotation [?].

We will analyze human performance of our task based on two measures, variable and signed error $[9,14]$. The variable error (variability between multiple trials) provides insight on precision, the signed error (mean of differences) on the accuracy of the rotation. In this work, each experimental condition will be repeated six times based on the experiment in [14]. We do not aim at analyzing the influence of the rotation angle on the error and therefore choose a constant target angle. Considering that humans perform better with horizontal or vertical orientations (oblique effect), we choose 90 degrees as target angle. Research about parallelism indicates that the perception of orientations is strongly biased by the hand-centered egocentric reference frame [15]. Hand orientations are insufficiently compensated and, therefore, influence the perceived orientation [13]. To reduce this confounding variable, all of our participants were instructed to take a defined upright body posture, with both of their arms and hands orthogonal to their body. We chose a precision grasp [1] to standardize the participants' movements and their posture.

\subsection{Notation}

In each experimental condition the participants were asked to grasp a threedimensional rotary knob at a particular position, so that a chosen local shape element (flat, round, edged) was located under the tips of the thumb and the index finger (see Fig. 2). We will denote all actions performed with the thumb and the index finger with $T$ and $I$, respectively. We will use $\mathbf{F}, \mathbf{R}$ and $\mathbf{E}$ to denote finger-independent actions with "flat", "round" and "edged" local shape elements, respectively. We denote individual experimental conditions for both fingers by combining the above notation into a tupel. For example, $\left(\mathbf{F}_{T}, \mathbf{R}_{I}\right)$ will be used to denote an experimental condition in which a flat surface is located under the thumb $\mathbf{F}_{T}$, and a round surface is located under the index finger $\mathbf{R}_{I}$ (see Fig. 2 for an overview).

\section{Methods}

\subsection{Participants}

Twenty-one sighted participants (16 males, 5 females) joined the study. They were all right-handed and none of them reported any haptic impairments. Their age varied between 19 and 36. All, except one participant who refused, were compensated for their time. The protocol was approved by the Bielefeld University Ethics Committee.

\subsection{Apparatus and Data Recording}

Here we use a novel apparatus called Twister, consisting of a cupboard with an integrated shaft on which a test object can be mounted (see Fig. 1, left). ${ }^{4}$

\footnotetext{
${ }^{4}$ https://ni.www.techfak.uni-bielefeld.de/node/3573
} 


\begin{tabular}{|c|c|c|c|c|}
\hline Thumb & Index finger & Notation & Rotary knob & Illustration \\
\hline flat & flat & $\left(F_{T}, F_{1}\right)$ & & \\
\hline flat & round & $\left(F_{T}, R_{1}\right)$ & & \\
\hline flat & edged & $\left(F_{T}, E_{l}\right)$ & & \\
\hline round & flat & $\left(R_{\mathrm{T}}, \mathrm{F}_{\mathrm{l}}\right)$ & & \\
\hline round & round & $\left(R_{T}, R_{l}\right)$ & & \\
\hline round & edged & $\left(R_{T}, E_{l}\right)$ & & \\
\hline edged & flat & $\left(E_{T}, F_{l}\right)$ & & \\
\hline edged & round & $\left(E_{T}, R_{l}\right)$ & & \\
\hline edged & edged & $\left(E_{T}, E_{1}\right)$ & & \\
\hline
\end{tabular}

Fig. 2. Overview of the nine experimental conditions. The rotary knob was grasped with thumb and index finger. Columns 1 and 2: Between the experimental conditions we varied the local shape elements which were grasped. Column 3: The notation for the experimental conditions. Column 4: Schematic illustration of the rotary knobs from the top view. The points, denote the position of the grasp during the rotation. Column 5: Illustration of the grasped local shape elements under the fingers. Participants grasped the rotary knobs from the top (see also Fig. 4).

Mounted knobs can be rotated either by the participant or controlled by the integrated direct-drive coreless electric motor (Maxon). The rotation induced by the participants can be performed with nearly zero resistance. The absolute shaft's orientation and, thus, the mounted rotary knob's orientation, is measured using a contactless digital magnetic hall encoder (AMS AS5048A). The sensor 
has a 360 degrees range and a resolution of 14 bit resulting in a step size of 0.022 degrees. By exploiting the skin conductance, Twister determines when the contact between a participant and the mounted object is established and how long it lasts. Twister measures the low current flowing from a grounded bracelet through a human hand into the conductive objects on the shaft $(5 \mathrm{~V}$ DC). The system is controlled by a PIC32 micro controller embedding a realtime motor control loop to achieve the desired angle deflections. The PIC32 communicates via USB with a host computer, which is equipped with a graphical control interface. The evaluation presented in this work is exclusively based on the measurement of the mounted objects' orientation.

For our experiment, we mounted four different shaped rotary knobs on the Twister's shaft (see Fig. 1, right). The rotary knobs are made of CNC-machined aluminium to allow the contact measurement. Their matte surface is sandblasted to disperse light reflections, so that the Twister can be used in combination with infrared-based motion tracking systems. The rotary knobs' diameter is $40 \mathrm{~mm}$ on the widest and $35 \mathrm{~mm}$ on the smallest position. Their height is $70 \mathrm{~mm}$, however, during the experiment participants grasped maximal the top $15 \mathrm{~mm}$ (see Fig. 4).

The experimental trials were also recorded with a high speed video camera (Basler, type piA640-210gc) from the top view. Additionally, 16 high speed infrared motion cameras (Vicon, MX3+ series) tracked 28 reflective markers placed on the right hand, arms and shoulders of the participants. The recordings of their 3D-trajectories will support analysis of hand posture and body orientation during the rotation task to be reported in future publications.

\subsection{Procedure and Task}

Participants signed an informed consent and filled a short questionnaire containing two tasks to examine their understanding of 90 degrees. In the first one, they had to draw an orthogonal line to a given line. In the second one, they were given a circle representing a rotary knob and a line to show the rotary knob's start orientation. Participants had to mark the rotary knob's end position after rotating an imaginary amount of 90 degrees counterclockwise. Afterwards, they had to read the task instructions, the reflective markers were attached and they were blindfolded.

During the trials they had to stand upright in front of Twister. Their right arm was in a horizontal orientation and both arms orthogonal to the body orientation (see Fig. 3). Participants performed the rotation task with the right hand, while the left hand rested on the table to maintain their body orientation. The height of the stage, on which the study participants were placed during the experiments, was adjusted to approximately reach a 90 degrees elbow orientation (see Fig. 3). However, no measures were taken to control this orientation during the experiment.

Within each trial, the procedure was as follows: In the beginning the right hand rested on the table. After the investigator verbally cued them to ready, they had to grasp the rotary knob with their thumb and index finger (see Fig. 4). In case a participant did not grasp at the right positions, the investigator corrected 

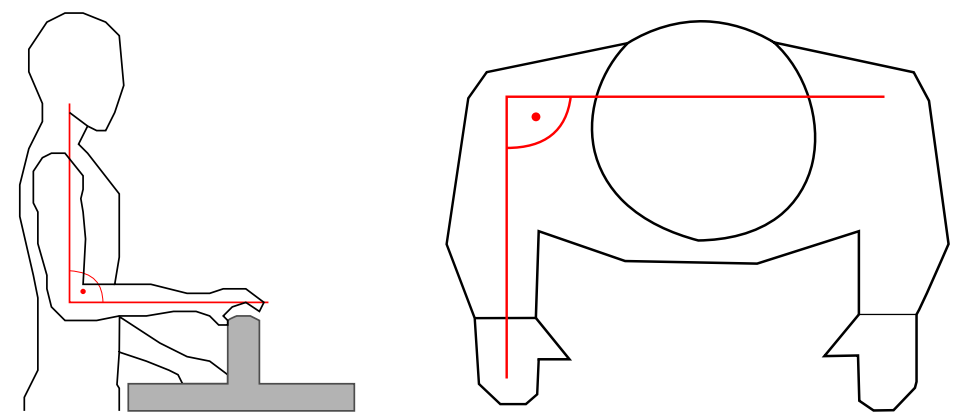

Fig. 3. The standardized body posture during the experimental trials.

their grip. The rotary knob was locked during this stage, in order to ensure the desired starting position in all trials, and to enable participants to grasp more easily without rotating the knob by accident through its movability. After this period the rotary knob was unlocked and participants had to rotate it 90 degrees counterclockwise. This rotation direction was chosen according to participant feedback of a pilot study that showed that this direction is easier with the right hand while sustaining the chosen grip. To be able to track the whole hand with motion tracking, participants were asked not to clench their fist (see Fig. 4 (right)). After finishing the rotation, they had to put their right hand again back to the starting position on the table.
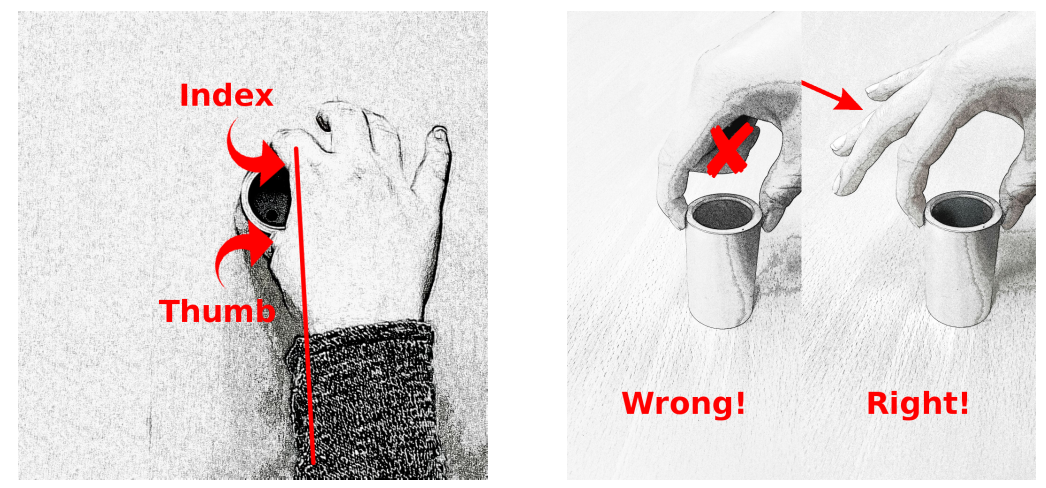

Fig. 4. Illustration of the correct grip. Participants had to position index finger and thumb in a line that was the elongation of their forearm. They had to avoid clenching their fist during the grasp and rotation phase.

\subsection{Experimental Design}

We compared three different local shape elements (round, edged and flat) and participants grasped the rotary knob with thumb and index finger. We chose a grasp with two fingers, as a grasp with an additional finger would have exponentiated the number of experimental conditions. This resulted in a 3 (shapes under 
the thumb) $\times 3$ (shapes under the index finger) within subjects design. The rotary knobs and their positions during the grasp are displayed in Fig. 2. Since we aimed at determining precision, we had to repeat each experimental condition six times. The experiment therefore consisted of 54 trials that took approximately one hour to complete. The trials were randomized for each participant individually in 6 blocks, with nine different experimental conditions each.

\subsection{Analysis}

For each trial, the relative rotation angle (in degrees) produced by the participants was extracted from the absolute angle data delivered by the Twister. The algebraic differences between the target angle of 90 degrees and the extracted rotation angles were computed. Based on the algebraic differences, two performance measures were calculated for each participant and each experimental condition: variable and signed error. The variable error is defined as the standard deviation of all six algebraic differences belonging to one experimental condition. Values closer to zero are associated with a higher precision. Signed error is calculated as the mean of the six algebraic differences. The estimated value can be either positive or negative, values closer to zero are associated with higher accuracy.

Mauchly's Test was conducted for both performance measures to test whether the assumptions of sphericity were violated. Afterwards a two-way repeated measures ANOVA was performed on both measures with the factors shapes under the thumb and shapes under the index finger. A significance level of $\alpha=0.05$ was chosen. For significant results post hoc tests with Bonferroni correction were conducted.

\section{$3 \quad$ Results}

\subsection{Variable Error}

The variable mean error was $M=11.58$ degrees ( $S D=7.81$ degrees). Fig. 5 (top) illustrates the variable error's mean for each experimental condition. The dotted line representing $\mathbf{F}_{I}$ conditions (flat shape under the index finger) had the smallest means of variable errors. The means of variable errors were the highest in $\mathbf{E}_{I}$ conditions (edged surface under the index finger) shown by the dashed line. This was also the same for the thumb: the means of variable errors condition $\mathbf{F}_{T}$ were the smallest, while the means of errors in the condition $\mathbf{E}_{T}$ were the highest. Based on Fig. 5, it seems that a flat shape led to smaller variable errors and therefore to a better precision. In contrast, an edged shape induces bigger variable errors, so the precision is worse. For each dot representing the variable error's mean in Fig. 5 (top) a boxplot representing the variable error's distribution is plotted in Fig. 5 (bottom). Comparing both figures reveals that the difference between the variable error's means in the conditions is small while the variable error's distribution is wide. The assumption of sphericity was not violated and, therefore, we conducted an ANOVA which did not show a significant difference between the conditions. 

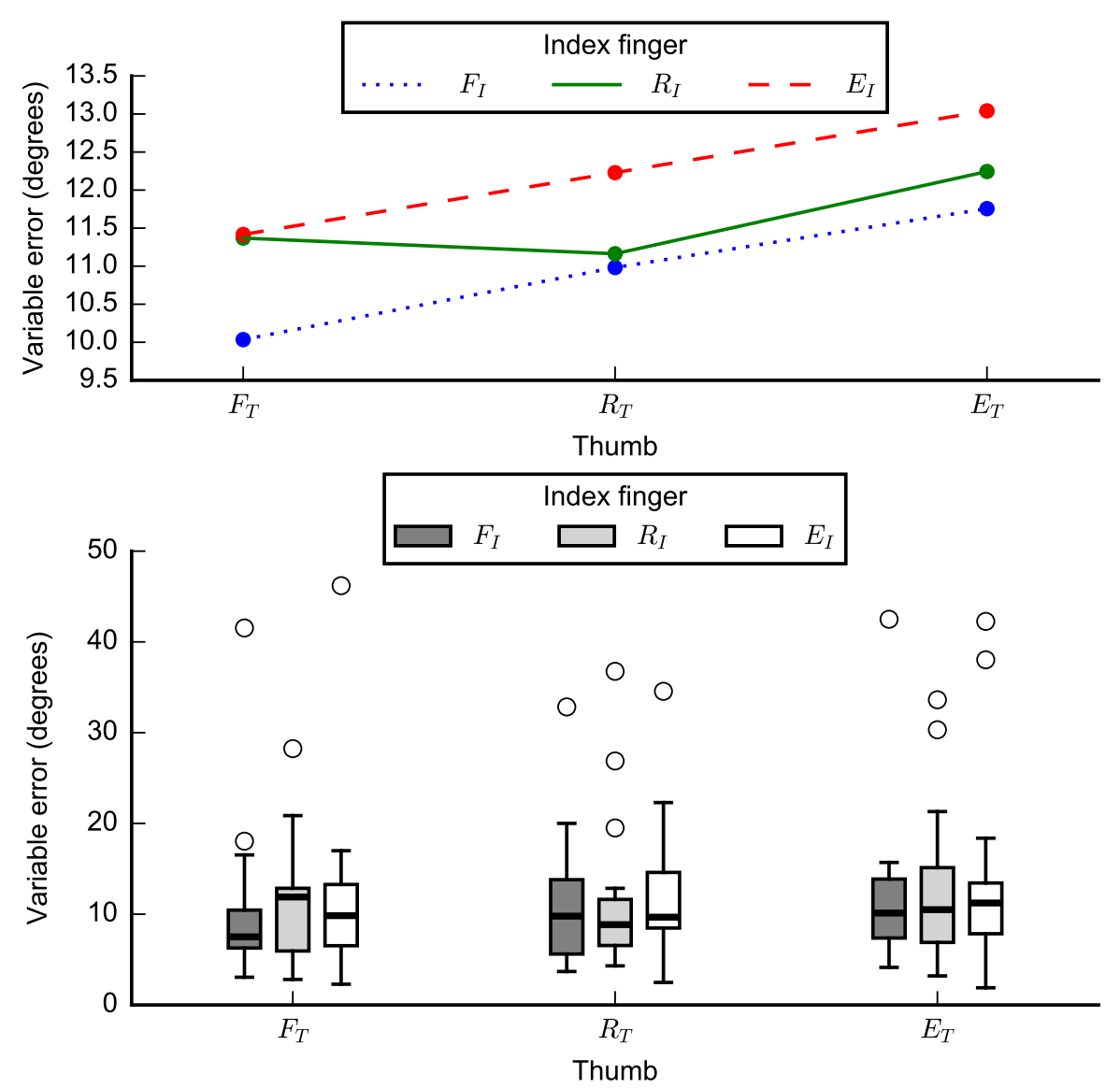

Fig. 5. Variable errors in the different experimental conditions. Top: Means of variable errors in the corresponding experimental conditions are shown on the $y$-axis. The factor shapes under the thumb is plotted on the $x$-axis, while the factor shapes under the index finger is shown via three different line styles. Each dot stands for a combination of both factors, which results in the nine experimental conditions. Bottom: Grouped boxplots show the distribution of variable errors in the nine experimental conditions. Each group of boxplots belongs to a specific shape under the thumb condition which is plotted on the $x$-axis. The color of the boxplots specifies the shape under the index finger condition. The variable error is plotted on the $y$-axis.

\subsection{Signed Error}

The mean of the signed error was $M=12.97$ degrees $(S D=24.59$ degrees). Fig. 6 (top) presents the means for all experimental conditions. In contrast to the variable errors, the means of the signed errors were the highest in the condition $\mathbf{R}_{I}$ (round shape under index finger). Consistent with the variable errors the means of signed errors were smallest in most of the conditions $\mathbf{F}_{I}$ (flat shape was under the index finger). Only the condition $\left(\mathbf{F}_{I}, \mathbf{R}_{T}\right)$ is an exception. Additionally, the figure shows that means of signed errors were higher in the condition $\mathbf{R}_{T}$ (round 


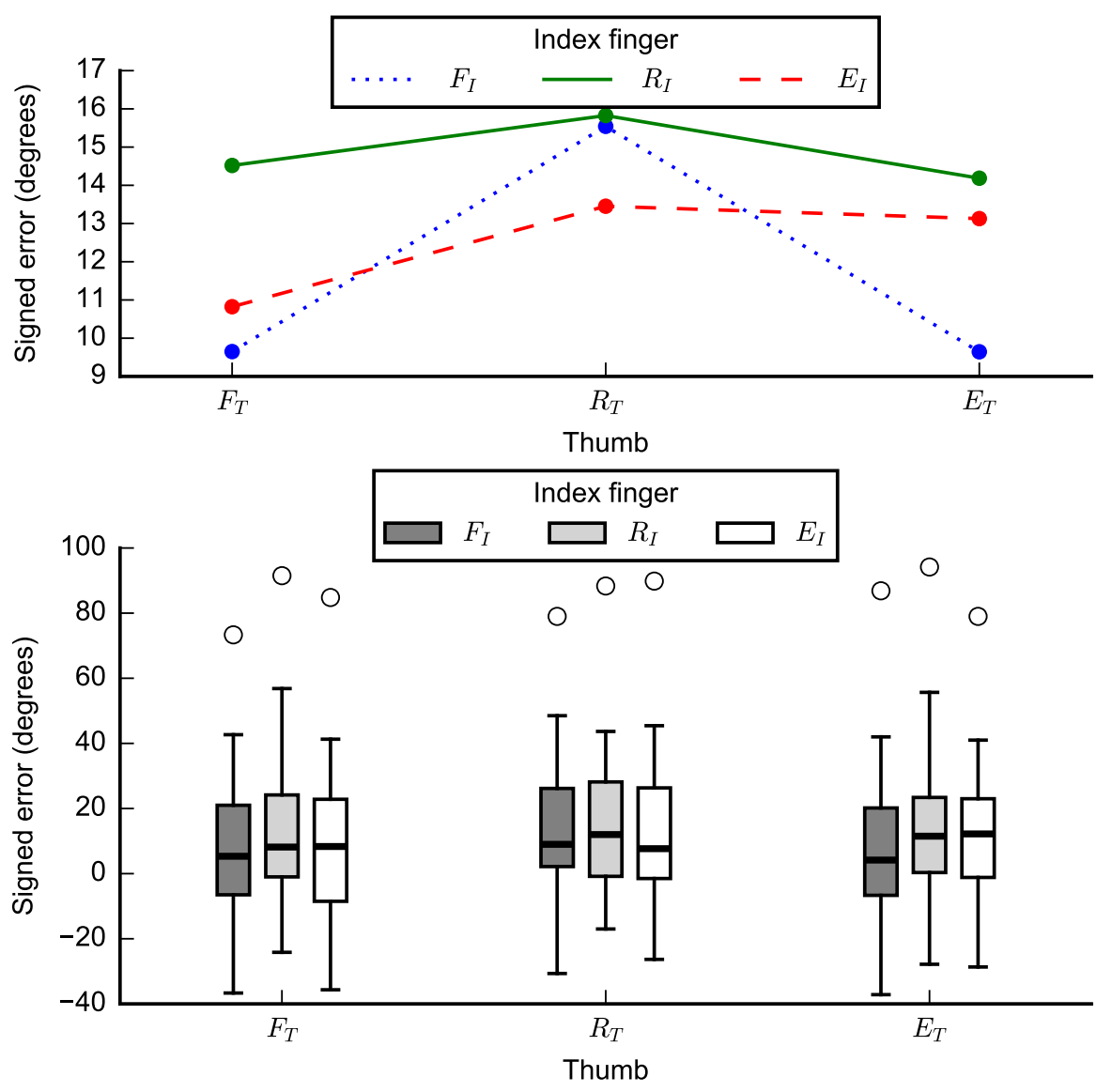

Fig. 6. Signed errors in all nine experimental conditions. See Fig. 5 for further explanations.

shape is under the thumb) than in the other two conditions $\mathbf{F}_{T}$ and $\mathbf{E}_{T}$. Fig. 6 (bottom) contains boxplots representing the signed error's distribution for each experimental condition. Just like the variable errors, this figure implies that the distribution of the signed errors is wide whereas the difference between the mean signed errors in the different conditions (see Fig. 6 (top)) is comparatively small.

Since the Mauchly-Test was not significant, we performed the ANOVA. Results showed a significant main effect for the first factor $T$, shape under the thumb $\left(F(2,40)=7.167, p=0.002, \eta_{\text {partial }}^{2}=0.262\right)$. Post hoc tests revealed that there is a significant difference between the round and the flat condition $\mathbf{R}_{T}$ and $\mathbf{F}_{T}(p=0.004)$, but no differences between all other conditions $\left(\mathbf{F}_{T}\right.$ and $\mathbf{E}_{T}$ : $p=1.000, \mathbf{E}_{T}$ and $\left.\mathbf{R}_{T}: p=0.053\right)$. This means that in case of a flat shape under the thumb the signed error was significantly smaller than when a round shape was under the thumb, as shown in the Fig. 6 . There was also a significant main effect for the second factor $I$, shape for the index finger $(F(2,40)=6.607$, 
$\left.p=0.003, \eta_{\text {partial }}^{2}=0.248\right)$. Post hoc tests were also significant for the difference between the flat and the round condition $\mathbf{F}_{T}$ and $\mathbf{R}_{T}(p=0.020)$, where the flat condition had smaller values. Results did not show significant differences for conditions $\mathbf{F}_{I}$ and $\mathbf{E}_{I}(p=0.691)$ and $\mathbf{E}_{I}$ and $\mathbf{R}_{I}(p=0.069)$. According to this, participants produced higher signed errors when they had a round, rather than a flat shape, under their index finger. This is reflected in Fig. 6.

Last, there was also a significant interaction between both factors $\left(F(4,80)=2.921, p=0.026, \eta_{\text {partial }}^{2}=0.127\right)$. This means, that the effect of the shapes is not an additive one. Fig. 6 (top) shows this phenomenon. In case of no interaction the dotted line representing $\left(\mathbf{F}_{I}\right)$ conditions would show the smallest signed errors independent of the shape on the thumb. However, results show that the mean signed error of the condition $\left(\mathbf{R}_{T}, \mathbf{F}_{I}\right)$ is higher than the mean signed error of condition $\left(\mathbf{R}_{T}, \mathbf{E}_{I}\right)$.

\subsection{Rotation Bias}

Since the mean signed error was greater than zero, we investigated whether participants rotated significantly more than 90 degrees. We analyzed whether the signed errors were significantly greater than zero, because a signed error of zero degrees represents a perfect rotation around 90 degrees.

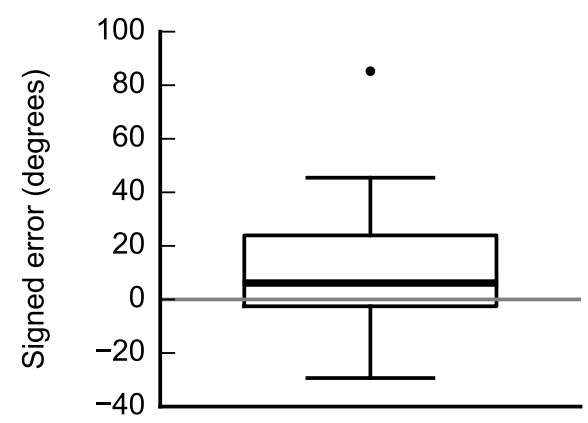

Fig. 7. The boxplot shows the distribution of participant-wise means of the signed errors. The line at zero degree stands for a perfect rotation around 90 degrees. Signed errors with positive values implies that participants rotated more than the desired 90 degrees.

We calculated the means of their signed errors for all participants. A boxplot containing these 21 values is shown in Fig. 7 . It illustrates that the median (the thick line) is higher than zero. We tested for normal distribution using a Shapiro-Wilk test, and as this assumption was not violated, we performed a one sampled, one-tailed t-test against zero. The chosen significance level was $\alpha=0.05$. The result was $t(20)=2.41, p=0.013$. According to this, participants rotated over all experimental condition more than 90 degrees. 


\section{Discussion}

We have investigated a task of performing a 90 degrees rotation with a two-finger precision grasp. Nine experimental conditions were tested to evaluate whether and how local shape elements located under the fingers while rotating influence the performance of the participants with regard to the different error measures. Our evaluation showed that the signed error is significantly influenced by the shape located under both fingers. Flat surface under both fingers yielded the best performance, round shape under both fingers yielded the worst performance. Based on the conducted work and the presented results, we presume that humans have a bias toward rotating too far.

In evaluation of variable error (see Section 3.1), no significant effect was found. According to our expectations, we have found a significant effect of shape elements on the signed error. For both the index finger and the thumb there was a significant difference between the signed error in the round and the flat condition, $\mathbf{R}$ and $\mathbf{F}$ respectively. Although the effect is present, we do not know what causes it. Both contact mechanics and proprioception may play a role and need to be investigated separately and in interaction. At least two hypotheses are possible. The contact mechanics may determine the error, with the round case possibly exhibiting a more complex dynamic compared to the flat case. Alternatively, interaction of proprioception and contact mechanics may result in a different strategy and determine the error w.r.t. the shapes $\mathbf{F}, \mathbf{E}$ or $\mathbf{R}$. We will analyze the recorded Vicon data to investigate whether there is a difference between performed strategies.

We found an unexpected increase of the signed error in the condition with round shape under thumb and flat shape under the index finger $\left(\mathbf{R}_{T}, \mathbf{F}_{I}\right)$. This may be due to the fact that the surface that had to be grasped with the index finger was not sufficiently wide and, therefore, additional unwanted shape cues influenced the performance.

Our evaluation result concludes that there exists a bias towards rotating too far, which applies to all experimental conditions. However, the shape seems to deliver additional orientational cues that intervene and affect the performance. Future work needs to investigate the reasons for this bias by e.g. evaluating a larger set of local object features varying at a small resolution, e.g. the angle in the edged conditions increasing from 90 to 180 degrees in 10 degrees steps. Additionally, further object features should be analyzed, whether they can improve performance and eliminate the bias.

\section{Acknowledgment}

This work was supported by the DFG Center of Excellence EXC 277: Cognitive Interaction Technology (CITEC) and the German-Japan Collaborative Research Program on Computational Neuroscience (RI 621/9-1). We would like to thank Risto Kõiva and his team for developing the Twister, Jan Anlauff for editing, and Christian Klarhorst for technical support and fruitful discussions. 


\section{References}

1. Feix, T., Pawlik, R., Schmiedmayer, H.B., Romero, J., Kragic, D.: A comprehensive grasp taxonomy. In: Robotics, science and systems: workshop on understanding the human hand for advancing robotic manipulation. vol. 2, pp. 2-3 (2009)

2. Fernández-Díaz, M., Travieso, D.: Performance in haptic geometrical matching tasks depends on movement and position of the arms. Acta psychologica 136(3), 382-389 (2011)

3. Flanders, M., Soechting, J.F.: Frames of reference for hand orientation. Journal of Cognitive Neuroscience 7(2), 182-195 (1995)

4. Gentaz, E., Badan, M., Luyat, M., Touil, N.: The manual haptic perception of orientations and the oblique effect in patients with left visuo-spatial neglect. NeuroReport 13(3), 327-331 (2002)

5. Gentaz, E., Baud-Bovy, G., Luyat, M.: The haptic perception of spatial orientations. Experimental brain research 187(3), 331 (2008)

6. Gentaz, E., Hatwell, Y.: Role of gravitational cues in the haptic perception of orientation. Attention, Perception, \& Psychophysics 58, 1278-1292 (1996)

7. Gentaz, E., Hatwell, Y.: The haptic oblique effect in the perception of rod orientation by blind adults. Perception \& Psychophysics 60(1), 157-167 (1998)

8. Gentaz, E., Hatwell, Y.: Role of memorization conditions in the haptic processing of orientations and the oblique effect. British Journal of Psychology 90(3), 373-388 (1999)

9. Howard, I.P.: Human visual orientation. John Wiley \& Sons (1982)

10. Kappers, A.M.L.: Large systematic deviations in the haptic perception of parallelity. Perception 28(8), 1001-1012 (1999)

11. Kappers, A.M.L.: Haptic perception of parallelity in the midsagittal plane. Acta psychologica 109(1), 25-40 (2002)

12. Kappers, A.M.L.: The contributions of egocentric and allocentric reference frames in haptic spatial tasks. Acta psychologica 117(3), 333-340 (2004)

13. Kappers, A.M.L., Viergever, R.F.: Hand orientation is insufficiently compensated for in haptic spatial perception. Experimental Brain Research 173(3), 407-414 (2006)

14. Luyat, M., Moroni, C., Gentaz, E.: The role of contextual cues in the haptic perception of orientations and the oblique effect. Psychonomic bulletin \& review 12(4), 760-766 (2005)

15. Van Mier, H.I.: Haptic perception of parallelity. Psychol Behav Sci 3, 212-221 (2014)

16. Volcic, R., Kappers, A.M.L., Koenderink, J.J.: Haptic parallelity perception on the frontoparallel plane: the involvement of reference frames. Attention, Perception, \& Psychophysics 69(2), 276-286 (2007) 